\title{
Optimizing cardiac CT angiography minimum detectable difference via Taguchi's dynamic algorithm, a V-shaped line gauge, and three PMMA phantoms
}

\author{
Lung-Fa Pan ${ }^{\mathrm{a}, \mathrm{b}}$, Yi-Hua Chen ${ }^{\mathrm{a}}$, Chun-Chieh Wang ${ }^{\mathrm{a}, \mathrm{c}}$, Bing-Ru Peng ${ }^{\mathrm{a}, \mathrm{d}}$, \\ Samrit Kittipayak ${ }^{\mathrm{e}, *}$ and Lung-Kwang Pan ${ }^{\mathrm{a}, *}$ \\ ${ }^{a}$ Department of Medical Imaging and Radiological Science, Central Taiwan University of Science and \\ Technology, Takun, Taichung, Taiwan \\ ${ }^{\mathrm{b}}$ Department of Cardiology, Taichung Armed Forces General Hospital, Taichung, Taiwan \\ ${ }^{\mathrm{c}}$ Puli Branch, Taichung Veterans General Hospital, Nantou, Taiwan \\ ${ }^{\mathrm{d}}$ Department of Radiology, Taichung Armed Forces General Hospital, Taichung, Taiwan \\ ${ }^{\mathrm{e}}$ Department of Radiological Technology, Faculty of Medical Technology, Mahidol University, Bangkok, \\ Thailand
}

\begin{abstract}
.
BACKGROUND: Radiologists widely use the minimum detectable difference (MDD) concept for inspecting the imaging quality and quantify the spatial resolution of scans.

OBJECTIVE: This study adopted Taguchi's dynamic algorithm to optimize the MDD of cardiac CT angiography (CTA) using a V-shaped line gauge and three PMMA phantoms (50, 70, and $90 \mathrm{~kg}$ ).

METHODS: The phantoms were customized in compliance with the ICRU-48 report, whereas the V-shaped line gauge was indigenous to solidify the cardiac CTA scan image quality by two adjacent peaks along the V-shaped slit. Accordingly, the six factors A-F assigned in this study were A (kVp), B (mAs), C (CT pitch), D (FOV), E (iDose), and F (reconstruction filter). Since each factor could have two or three levels, eighteen groups of factor combinations were organized according to Taguchi's dynamic algorithm. Three welltrained radiologists ranked the CTA scan images three times for three different phantoms. Thus, $27(3 \times 3 \times 3)$ ranked scores were summed and averaged to imply the integrated performance of one specific group, and eventually, 18 groups of CTA scan images were analyzed. The unique signal-to-noise ratio $(\mathrm{S} / \mathrm{N}, \mathrm{dB})$ and sensitivity in the dynamic algorithm were calculated to reveal the true contribution of assigned factors and clarify the situation in routine CTA diagnosis.

RESULTS: Minimizing the cross-interactions among factors, the optimal factor combination was found to be as follows: A $(100 \mathrm{kVp}), \mathrm{B}(600 \mathrm{mAs}), \mathrm{C}$ (pitch $0.200 \mathrm{~mm}$ ), D (FOV $280 \mathrm{~mm}$ ), E (iDose 5), and F (filter XCA). The respective MDD values were 2.15, 2.32, and $1.87 \mathrm{~mm}$ for 50, 70, and $90 \mathrm{~kg}$ phantoms, respectively. The MDD of the $90 \mathrm{~kg}$ phantom had the most precise spatial resolution, while that of the $70 \mathrm{~kg}$ phantom was the worst.

CONCLUSION: The Taguchi static and dynamic optimization algorithms were compared, and the latter's superiority was substantiated.
\end{abstract}

Keywords: Taguchi dynamic, phantom, CT angiography, resolution

\footnotetext{
${ }^{*}$ Corresponding authors: Samrit Kittipayak, Department of Radiological Technology, Faculty of Medical Technology, Mahidol University, Bangkok, Thailand. E-mail: samrit.kit@mahidol.ac.th. Lung-Kwang Pan, Department of Medical Imaging and Radiological Science, Central Taiwan University of Science and Technology, Takun, Taichung, Taiwan. E-mail: 1kpan@ctust.edu.tw.
} 


\section{Introduction}

This study optimized the minimum detectable difference (MDD) of cardiac CT angiography (CTA) using three (50, 70, and $90 \mathrm{~kg}$ ) PMMA phantoms and a V-shaped line gauge according to Taguchi's dynamic algorithm. Radiologists widely use the MDD concept for inspecting the imaging quality and quantify the spatial resolution of scans [1-5]. The MDD advantage over the conventional line pair per $\mathrm{cm}$ approach can be solidified in many aspects because MDD accounts for the distance between two adjacent peaks from SPECT (single-photon emission computed tomography) profile and the accompanied FWHM (full width of half maximum) of peaks.

The imaging quality of scans in medical facilities, such as X-ray, cardiac bi-plane X-ray, CT, CTA, mammography, and a gamma camera, is vital for the accurate diagnosis. Therefore, multiple techniques have been introduced to upgrade the spatial resolution, compromising the high quality of the acquired images and an acceptable exposed dose required for their provision [6-13]. Taguchi's optimization analysis became an assessable technique in providing quantified data for optimization due to its userfriendly and reliable realization. In contrast, Taguchi's dynamic algorithm is seldom applied in clinical approaches because the dynamic scenario has its inherent limitation in empirical preset. Specifically, the surveyed target (either a phantom or a gauge) needs to be replaced to compose the dynamic scenario. The target replacement via the dynamic algorithm is mandatory in evaluating the quality sensitivity [14], which is a required index to justify the dynamic quality characteristic.

In this study, the V-shaped gauge was placed into three PMMA phantoms with various geometrical sizes and weights, namely 50,70, or $90 \mathrm{~kg}$ to evaluate the MDD in clinical cardiac CTA scans. The V-shaped gauge had to be scanned 18 times via Taguchi's dynamic algorithm to collect the images for optimizing them via conversion by MATLAB and plotting by the ORIGIN default program to obtain the MDD. The unique quality characteristic of sensitivity in the dynamic algorithm was interpreted for different scenarios to satisfy the special consideration of the cardiac CTA technique.

\section{Materials and methods}

\subsection{Orthogonal array}

The unique feature of Taguchi's algorithm is that it not only optimizes assigned factors from a particular dataset but also identifies and ranks factors influencing the main variable. The six factors for cardiac CTA scan protocol were $\mathrm{kVp}, \mathrm{mAs}$, pitch (the distance of table travel per a $360^{\circ}$ gantry rotation ratio to the whole thickness of all acquired slices simultaneously), FOV (field of view, $\mathrm{mm}$ ), iDose (a reconstruction algorithm to improve the image quality through artifact prevention and increased spatial resolution at low dose $)$ and reconstruction filter type [14]. In total, $486(2 \times 3 \times 3 \times 3 \times 3 \times 3)$ combinations were analyzed since each of the six factors was defined into three or two levels. Adopting the Taguchi's algorithm, the samples could be organized into only eighteen groups, which were concluded results with same confident level as those obtained via the conventional and more thorough optimization processes [15]. Table 1 presents a standard $\mathrm{L}_{18}\left(2^{1} \times 3^{5}\right)$ orthogonal array suggested by Taguchi; the numbers in each column indicate the practical arrangements or factor's levels for the individual factors (A-F). The assigned six factors, namely (A) kVp, (B) mAs, (C) pitch, (D) FOV, (E) iDose, and (F) reconstruction filter, are listed in Table 2.

\subsection{Taguchi's dynamic algorithm}

Unlike the static preset in Taguchi's optimization, the dynamic one is reputed for its strong flexibility in 
Table 1

Taguchi suggests the standard $\mathrm{L}_{18}\left(2^{1} \times\right.$ $3^{5}$ ) orthogonal array; the numbers in each column indicates the practical layouts (or levels) of the specific factor (A-F)

\begin{tabular}{ccccccc}
\hline Group & \multicolumn{7}{c}{ Factor } \\
\cline { 2 - 6 } & $\mathrm{A}$ & $\mathrm{B}$ & $\mathrm{C}$ & $\mathrm{D}$ & $\mathrm{E}$ & $\mathrm{F}$ \\
\hline 1 & 1 & 1 & 1 & 1 & 1 & 1 \\
2 & 1 & 1 & 2 & 2 & 2 & 2 \\
3 & 1 & 1 & 3 & 3 & 3 & 3 \\
4 & 1 & 2 & 1 & 1 & 2 & 2 \\
5 & 1 & 2 & 2 & 2 & 3 & 3 \\
6 & 1 & 2 & 3 & 3 & 1 & 1 \\
7 & 1 & 3 & 1 & 2 & 1 & 3 \\
8 & 1 & 3 & 2 & 3 & 2 & 1 \\
9 & 1 & 3 & 3 & 1 & 3 & 2 \\
10 & 2 & 1 & 1 & 3 & 3 & 2 \\
11 & 2 & 1 & 2 & 1 & 1 & 3 \\
12 & 2 & 1 & 3 & 2 & 2 & 1 \\
13 & 2 & 2 & 1 & 2 & 3 & 1 \\
14 & 2 & 2 & 2 & 3 & 1 & 2 \\
15 & 2 & 2 & 3 & 1 & 2 & 3 \\
16 & 2 & 3 & 1 & 3 & 2 & 3 \\
17 & 2 & 3 & 2 & 1 & 3 & 1 \\
18 & 2 & 3 & 3 & 2 & 1 & 2 \\
\hline
\end{tabular}

Table 2

The six factors of cardiac CTA scan protocol, every factor having two or three levels as recommended by the Taguchi $\mathrm{L}_{18}$ orthogonal array

\begin{tabular}{llll}
\hline \multicolumn{1}{c}{ Factor } & Level 1 & Level 2 & Level 3 \\
\hline (A) $\mathrm{kVp}$ & 100 & 120 & \\
(B) $\mathrm{mAs}$ & 600 & 700 & 800 \\
(C) Pitch (mm) & 0.126 & 0.150 & 0.200 \\
(D) FOV (mm) & 220 & 250 & 280 \\
(E) iDOse & 3 & 4 & 5 \\
(F) Reconstruction Filter & XCC & XCB & XCA \\
\hline
\end{tabular}

satisfying various conditions in a clinical environment. The unique definition of sensitivity $(\beta)$ allows one to correlate the expectation value, i.e., signal-to-noise ratio versus the main variable. The latter may involve various PMMA phantom weights defined in this study or exposed radioactive doses [8,13]. Nevertheless, the $\beta$ parameter is preset via "higherthe-better" criterion to intensify good quality characteristics in the conventional robust designation. However, $\beta$ was redefined as "lowerthebetter" to reduce the sensitivity of phantom's weight in this study and reduced to

$$
\begin{aligned}
& \beta=\frac{\text { ranking in groups }}{\text { phantom weight }}=\frac{Y}{X}=\frac{\sum_{j=1}^{m} \sum_{i=1}^{n} x_{i j} y_{j}}{\sum_{j=1}^{m} \sum_{i=1}^{n} x_{i j}^{2}} \\
& \text { stdev }=\sqrt{\frac{\sum_{j=1}^{m} \sum_{i-1}^{n}\left(y_{j}-\beta x_{i j}\right)^{2}}{(n \times m)-1}} \\
& S / N(\mathrm{~dB})=-10 \log \left(\text { stdev }^{2} \cdot \beta^{2}\right)
\end{aligned}
$$

where $\beta$ is the sensitivity, and $\mathrm{Y}$ is the ranking of the V-shaped line gauge in 18 groups for the specific 


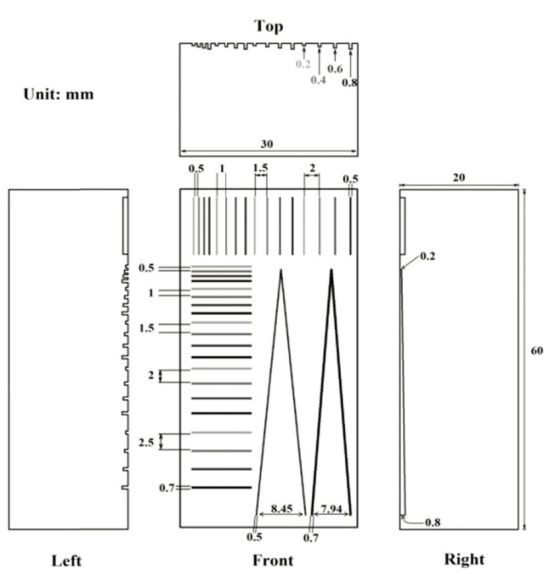

A

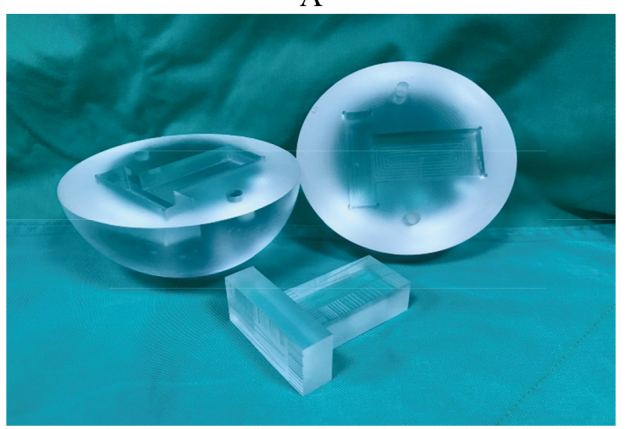

$\mathrm{C}$

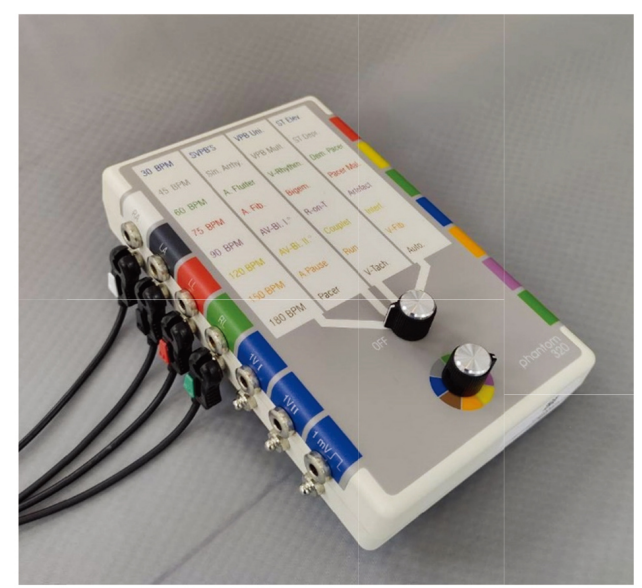

B

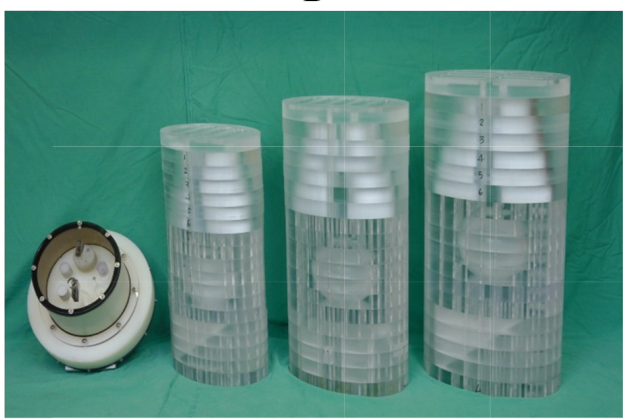

D

Fig. 1. (A) implies a special-made V-shaped line gauge with $20 \mathrm{~mm}$-high, $60 \mathrm{~mm}$-long, and $30 \mathrm{~mm}$-wide cuboid. In contrast, the two V-shaped lines had 0.5 or 0.7 widths, and the slit depth changed from $0.2 \mathrm{~mm}$ in the top to $0.8 \mathrm{~mm}$ in the bottom. The quantified survey focused on the left V-shaped line with a $0.5 \mathrm{~mm}$ width to justify the MDD in this measurement, (B) the commercial 320 ECG simulator was adopted to synchronize the CTA scanning frequency and simulated 60 BPM (beats per min.), (C) the tightly-composed cardiac ball with two vertical and horizontal V-shaped line gauges, and (D) three cardiac balls were placed into the 50, 70, and $90 \mathrm{~kg}$ PMMA phantoms, respectively.

phantom weight (i.e., 50, 70, or $90 \mathrm{~kg}$ ). Here, stdev is the standard deviation of the repeated ranking in obtaining the gauge ranks, averaged over $n$ (reviewers $\times$ repeated times) rankings for all $m$ weights. In this study, $n=9(3 \times 3)$ and $m=3$. Taguchi proposed the signal-to-noise ratio $(\mathrm{S} / \mathrm{N}, \mathrm{dB})$ to integrate the functional performance and quantify the effect of beta or stdev. A high $\mathrm{S} / \mathrm{N}$ indicates a low stdev or beta in the practical survey, which is preferable to satisfy the aim of this study.

\subsection{A V-shaped line gauge and PMMA phantoms}

Figure 1A depicts a specially made V-shaped line gauge with $20 \mathrm{~mm}$-high, $60 \mathrm{~mm}$-long, and $30 \mathrm{~mm}$ wide cuboid. As shown, the two V-shaped lines had 0.5 or 0.7 widths, and the slit depth gradually changed from $0.2 \mathrm{~mm}$ in the top to $0.8 \mathrm{~mm}$ in the bottom. Specifically, the top two lines were preset to barely contacted each other with a shallow slit of $0.2 \mathrm{~mm}$ depth only. In contrast, the gap between the two lines became $8.45 \mathrm{~mm}$ (for the $0.5 \mathrm{~mm}$ wide slit) or $7.94 \mathrm{~mm}$ (for the $0.7 \mathrm{~mm}$-wide slit) in $0.8 \mathrm{~mm}$-deep at the bottom. The quantified survey focused on the left $\mathrm{V}$-shaped line with a $0.5 \mathrm{~mm}$ width to solidify the MDD in this measurement, (B) A commercial 320 ECG simulator was adopted to synchronize CTA scanning 


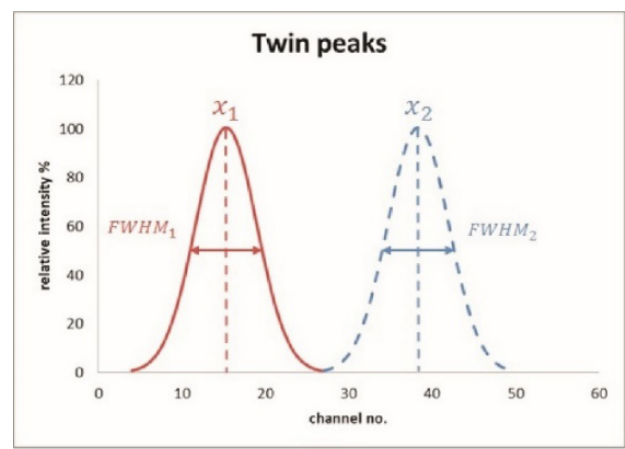

(A)

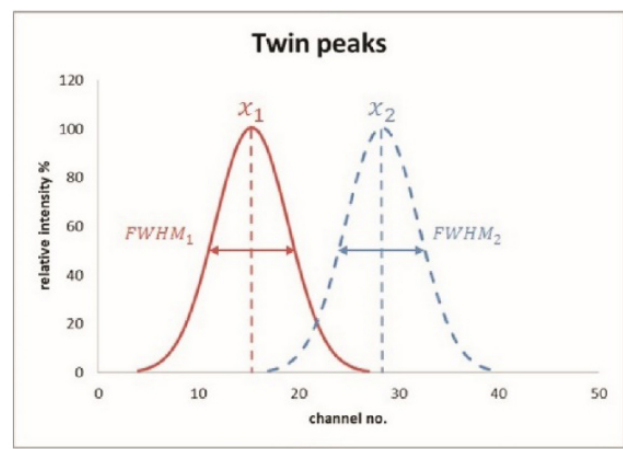

(B)

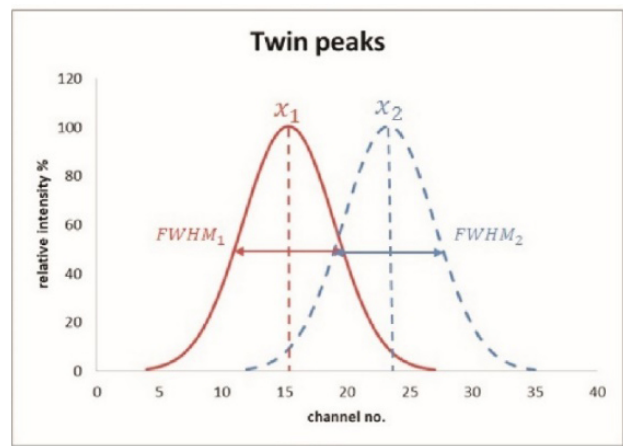

(C)

Fig. 2. (A) two peaks were recognized as fully separated; (B) two peaks scarcely passed the Student's $t$-test identification, while in $(C)$, the differentiation failed because the two peaks were too close to each other.

frequency and the simulated 60 BPM (beats per min.), (C) the tightly-composed cardiac ball with two vertical and horizontal V-shaped line gauges and (D) three cardiac balls were placed into the 50, 70, and $90 \mathrm{~kg}$ PMMA phantoms, respectively. The PMMA phantoms were revised according to the original ICRU-48 report [16]. In addition, a commercial phantom (model 4550-122-01471) for Philip Brilliance CT (model 128-sliced Philips Ingenuity) was also put aside for size comparing.

\subsection{The MDD definition and application}

The minimum detectable distance (MDD) between two peak centers was determined via the revised Student's $t$-test equation with a constant multiplication factor of 1.96, demonstrating two peak centers $X_{1}$ and $X_{2}$ deviated divergent enough to create a 95\% confident level of success [17]:

$$
\left|X_{1}-X_{2}\right| \geqslant 1.96 \times \sqrt{\left(\frac{F W H M_{1}}{2}\right)^{2}+\left(\frac{F W H M_{2}}{2}\right)^{2}},
$$

where $X_{1}$ and $X_{2}$ is the center of peaks 1 or 2, whereas FWHM is defined as full width at half the maximum of the specific peak. The MDD among line pairs was proposed to quantify the two overlappedpeak profiles that were collected from a tangent slice of a $0.5 \mathrm{~mm}$-deep V-shaped slit with two neighboring peaks, followed the various confident levels of success as implied in Eq. (4).

The MDD evaluation from two adjacent peaks via the specific V-shaped slit was performed by a gradual 
changing of the valley edge of two peaks and a quantitative assessment of the originally designed edge width. Unlike V-shaped water phantoms used in nuclear medicine examinations [18], the $0.5 \mathrm{~mm}$-deep V-shaped slit (cf. Fig. 1A) provided the finest edge between line pairs to $\sim 0.5 \mathrm{~mm}$ in a $0.2 \mathrm{~mm}$-deep slit, exceeding the distinguishable ability of average CT scan facilities. Therefore, various confidence levels of success can be assigned for the MDD assessment. Small MDD values of CT scan images imply a high distinguishable capability in cardiac artery or vein image analyses. As shown in Fig. 2A, two peaks were recognized as fully separated; (B) two peaks scarcely passed the Student's $t$-test identification, while in (C), the differentiation failed because the two peaks were too close to each other. This approach advantage over the qualitative judgment of radiologists is that the latter can be biased by the human factor, while the standard of failing or passing the revised Student's $t$-test are purely quantitative, fully based on the calculation results, and thus provide a more accurate and robust MDD assessment via quantified calculations [3-5].

\subsection{Cardiac CTA scan and ranking}

The imaging quality of the cardiac CT scan protocol was simulated by the V-shaped line gauge, as shown in Fig. 1A. To survey the gauge images' quality, each slit was infused contrast media solution $(\sim 3$ c.c. Optiray ${ }^{\circledR} 320$, a $68 \%$ Ioversol injection) and dyed with dark blue ink to pretend the real CT scan for the patient cardiac syndrome. Other factors for CT spiral rotation scanning were preset as follows: a spiral rotation time of $0.4 \mathrm{~s} / \mathrm{roll}$, a $400 \mathrm{~mm}$ collimator, and a slice thickness of $0.9 \mathrm{~mm}$ [4]. According to a 128-slice CT lung scan survey earlier conducted at Taichung Armed Forces General Hospital, Taiwan, the CT scan protocol underwent a minor revision for the 128-sliced Philips Ingenuity located at the above hospital. Three viewers ranked the scanned V-shaped gauge images from 18 groups with three repeated judgments under the double-blinded principle. Thus, every viewer had to check a total number of 162 images $(3 \times 3 \times 18=162)$ before the data analysis and further optimization.

\section{Results}

\subsection{Taguchi's optimization}

Figure 3 shows typical V-shaped line gauge images from cardiac CTA scans of 18 groups in the $70 \mathrm{~kg}$ PMMA phantom Since those obtained via 50 or $90 \mathrm{~kg}$ ones were quite similar, they were omitted for brevity. Some of them had low contrast ratios, being too bright or too dark to identify the V-shape. Table 3 lists the raw data obtained from the practical survey of CTA images. Each viewer ranked the 18 images three times for three different phantom sizes; thus, a total of $486(3 \times 3 \times 3 \times 18=486)$ ranking results were collected and statistically processed Beta, stdev, and S/N values derived via Eqs (1)-(3) are also included in the last three columns of Table 3. Furthermore, the fish-bone plots of various factors were constructed via Taguchi's dynamic algorithm [4,5]. As seen in Fig. 4, beta, stdev, and S/N exhibited strong fluctuations. Theoretically, low beta and stdev values and high $\mathrm{S} / \mathrm{N}$ provide the best expectation. Therefore, despite strong cross-interactions among factors, the preliminary suggestion (cf. Fig. 4) comprised A2 (120 kVp), B1 (600 mAs), C1 (pitch 0.126 mm), D3 (FOV $280 \mathrm{~mm}$ ), E3 (iDose 5), and F3 (filter XCA). Upon minimizing the cross-interactions among factors, the final optimal combination of factors became A1 (100 kVp), B1 (600 mAs), C3 (pitch 0.200 mm), D3 (FOV 280 mm), E3 (iDose 5), and F3 (filter $\mathrm{XCA}$ ). 


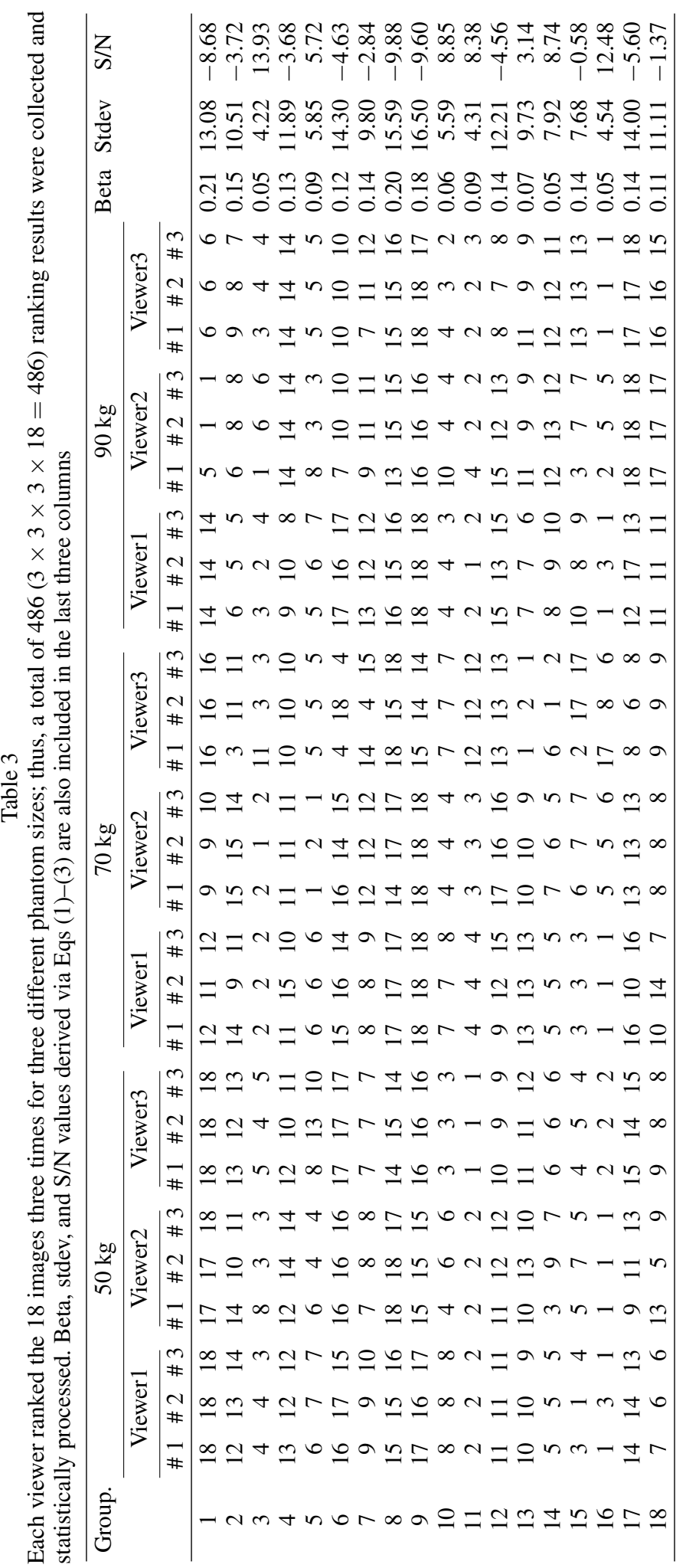




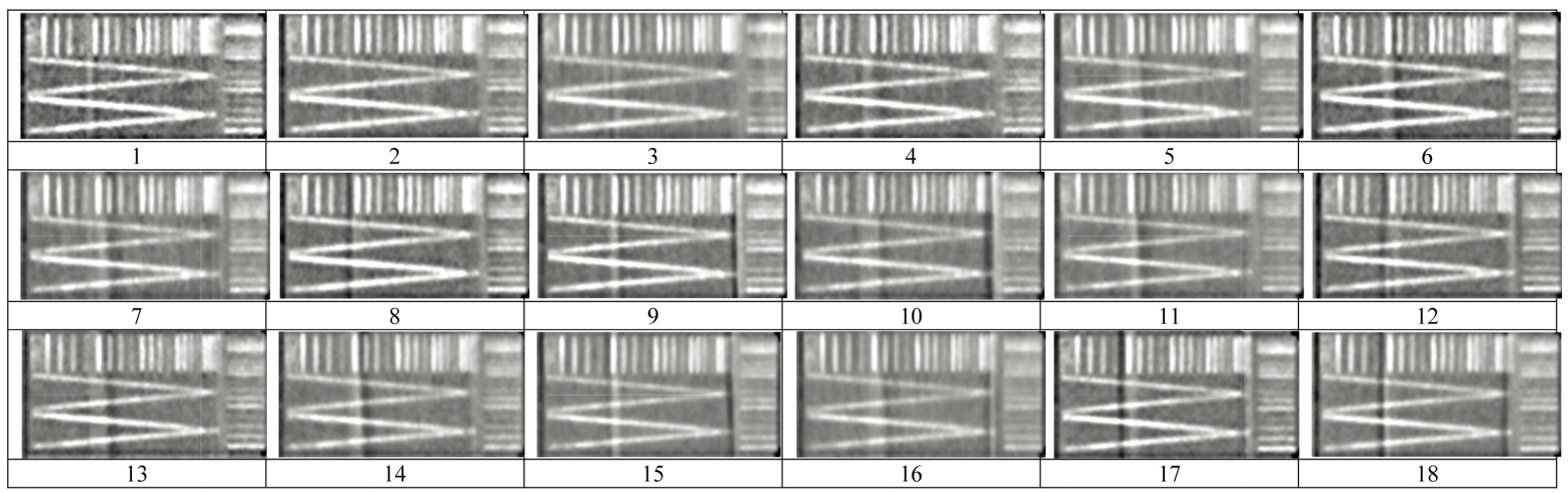

Fig. 3. Typical V-shaped line gauge images from cardiac CTA scans of 18 groups in the $70 \mathrm{~kg}$ PMMA phantom. Since those obtained via 50 or $90 \mathrm{~kg}$ ones were quite similar, they were omitted for brevity.

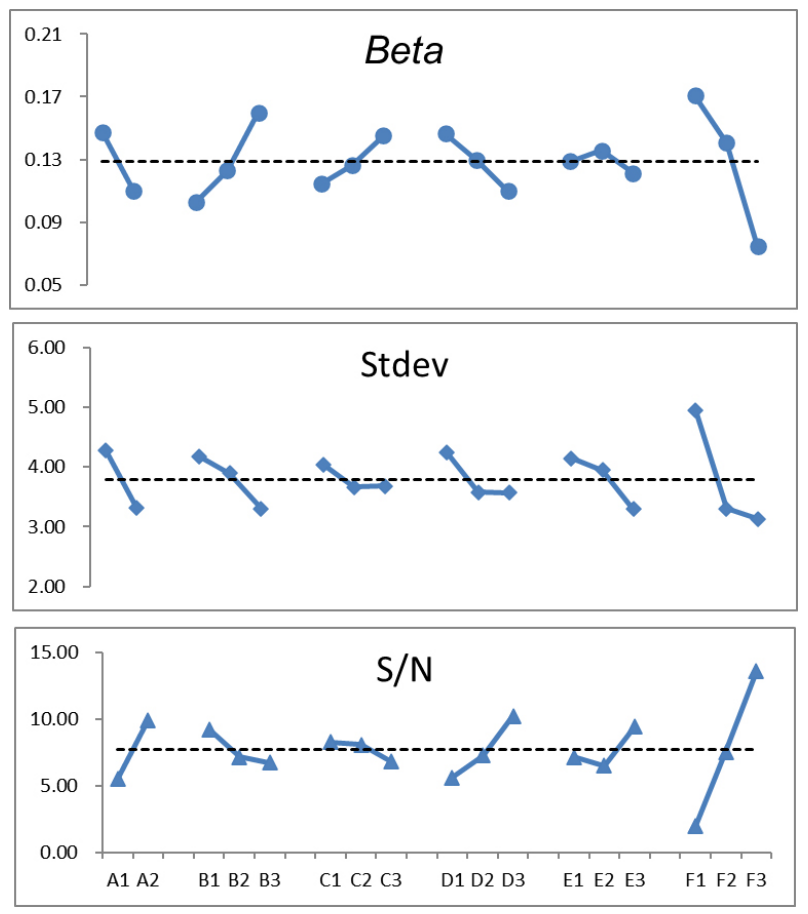

Fig. 4. The beta, stdev, and S/N exhibited strong fluctuations. Theoretically, low beta and stdev values and high S/N provide the best expectation.

\subsection{MDD of PMMA phantoms with various weights}

Table 4 shows the revised Student's $t$-test results for MDD derived via 50, 70, $90 \mathrm{~kg}$ PMMA phantoms. The optimal cardiac CTA scan factor combination was obtained, revealing the following trend: the right side's peaks failed the revised Student's $t$-test, while the left side's adjacent two peaks successfully passed it. The derived MDD values were $2.15,2.32$, and $1.87 \mathrm{~mm}$ for 50,70 , and $90 \mathrm{~kg}$ phantoms, respectively. The last one had the most precise spatial resolution, while that of $70 \mathrm{~kg}$ was the worst. The geometrical size/weight of the phantom significantly affected the image quality. Thus, a more precise 
Table 4

The revised Student's $t$-test results for MDD derived via 50, 70, $90 \mathrm{~kg}$ PMMA phantoms. The right side's peaks failed the revised Student's $t$-test, while the left side's adjacent two peaks successfully passed it

\begin{tabular}{|c|c|c|c|c|}
\hline MDD & & \multicolumn{3}{|c|}{$\left|\mathbf{X}_{1}-\mathbf{X}_{2}\right| \geqslant 1.96 \times \sqrt{\left(\frac{\text { FWHM }_{1}}{2}\right)^{2}+\left(\frac{\text { FWHM }_{2}}{2}\right)^{2}}$} \\
\hline $\begin{array}{l}50 \mathrm{~kg} \\
2.15 \mathrm{~mm}\end{array}$ & Pass & $\begin{array}{l}|18-30| \geqslant 1.96 \times \sqrt{\left(\frac{9}{2}\right)^{2}+\left(\frac{8}{2}\right)^{2}} \\
|-12| \geqslant 1.96 \times \sqrt{20.25+16} \\
12 \geqslant 11.8\end{array}$ & Fail & $\begin{array}{l}|19-28| \geqslant 1.96 \times \sqrt{\left(\frac{9}{2}\right)^{2}+\left(\frac{7}{2}\right)^{2}} \\
|-9| \geqslant 1.96 \times \sqrt{20.25+12.25} \\
9 \leqslant 11.17\end{array}$ \\
\hline $\begin{array}{l}70 \mathrm{~kg} \\
2.32 \mathrm{~mm}\end{array}$ & Pass & $\begin{array}{l}|11-18| \geqslant 1.96 \times \sqrt{\left(\frac{4}{2}\right)^{2}+\left(\frac{5}{2}\right)^{2}} \\
|-7| \geqslant 1.96 \times \sqrt{4+6.25} \\
7 \geqslant 6.28\end{array}$ & Fail: & $\begin{array}{l}|12-18| \geqslant 1.96 \times \sqrt{\left(\frac{5}{2}\right)^{2}+\left(\frac{5}{2}\right)^{2}} \\
|-6| \geqslant 1.96 \times \sqrt{6.25+6.25} \\
6 \leqq 6.93\end{array}$ \\
\hline $\begin{array}{l}90 \mathrm{~kg} \\
1.87 \mathrm{~mm}\end{array}$ & Pass & $\begin{array}{l}|23-34| \geqslant 1.96 \times \sqrt{\left(\frac{7}{2}\right)^{2}+\left(\frac{8}{2}\right)^{2}} \\
|-11| \geqslant 1.96 \times \sqrt{12.25+16.25} \\
11 \geqslant 10.42\end{array}$ & Fail: & $\begin{array}{l}|23-33| \geqslant 1.96 \times \sqrt{\left(\frac{8}{2}\right)^{2}+\left(\frac{9}{2}\right)^{2}} \\
|-10| \geqslant 1.96 \times \sqrt{16+20.25} \\
10 \leqq 11.8\end{array}$ \\
\hline
\end{tabular}

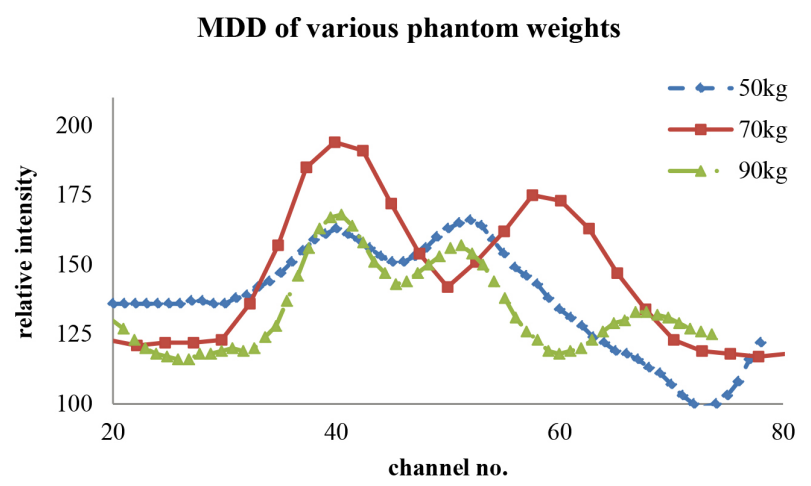

Fig. 5. The line-cut SPECT profile from the V-shaped line gauge for various phantom weights that just passed the Student's $t$-test.

factor combination of cardiac CTA had to be found according to the various phantom weights to ensure the best image quality. Specifically, Fig. 5 depicts the line-cut SPECT profile from the V-shaped line gauge for various phantom weights that just passed the Student's $t$-test. The SPECT data were converted via the MATLAB default software in this study. A too-large FWHM of the two peaks from the $70 \mathrm{~kg}$ phantom's SPECT profile deteriorated the MDD and reduced the spatial image resolution.

\section{Discussion}

\subsection{The ANOVA analytical result}

Static Taguchi's optimization requires the ANOVA analysis to discriminate dominant and minor factors via the variance analysis. In contrast, for dynamic Taguchi's one, the ANOVA is optional because the integrated performance is dominated by the unique quality characteristic of dynamic analysis, namely sensitivity [15]. However, by checking the sum of squared variances for various phantom weights, one can get a deeper insight into the fluctuation patterns. Consider the following parameters:

$$
S S_{\text {Total }}=\left[\sum_{i=1}^{n} \sum_{j=1}^{r} y_{i j}^{2}\right]-n \times r \times \overline{\bar{y}}^{2}
$$


Table 5

A significantly different distribution of $S S_{\text {Factor }}$ at various phantom weights: parameter $S S_{\text {error }}$ in the $70 \mathrm{~kg}$ phantom analysis reached $40 \%$, indicating an enormous discrepancy in viewers' ranking results, whereas its values in other weights were $7.8 \%$ for $50 \mathrm{~kg}$ and $20 \%$ for $90 \mathrm{~kg}$

\begin{tabular}{|c|c|c|c|c|c|c|c|}
\hline \multirow[t]{2}{*}{ Factor } & \multirow[t]{2}{*}{ DOF } & \multicolumn{2}{|c|}{$50 \mathrm{~kg}$} & \multicolumn{2}{|c|}{$70 \mathrm{~kg}$} & \multicolumn{2}{|c|}{$90 \mathrm{~kg}$} \\
\hline & & SS & Contri $\%$ & SS & Contri \% & SS & Contri \% \\
\hline A & 1 & 1200.50 & $27.53 \%$ & 352.60 & $8.09 \%$ & 53.39 & $1.22 \%$ \\
\hline B & 2 & 74.04 & $1.70 \%$ & 400.70 & $9.19 \%$ & 1217.37 & $27.92 \%$ \\
\hline $\mathrm{C}$ & 2 & 12.33 & $0.28 \%$ & 79.15 & $1.82 \%$ & 381.59 & $8.75 \%$ \\
\hline D & 2 & 184.11 & $4.22 \%$ & 194.70 & $4.47 \%$ & 213.37 & $4.89 \%$ \\
\hline E & 2 & 0.48 & $0.01 \%$ & 198.04 & $4.54 \%$ & 5.59 & $0.13 \%$ \\
\hline $\mathrm{F}$ & 2 & 2481.33 & $56.90 \%$ & 1112.11 & $25.50 \%$ & 1268.04 & $29.08 \%$ \\
\hline Others & 6 & 67.04 & $1.54 \%$ & 279.20 & $6.40 \%$ & 334.04 & $7.66 \%$ \\
\hline Error & 144 & 340.67 & $7.81 \%$ & 1744.00 & $40.00 \%$ & 887.11 & $20.34 \%$ \\
\hline Total & 161 & 4360.50 & $100.00 \%$ & 4360.50 & $100.00 \%$ & 4360.50 & $100.00 \%$ \\
\hline
\end{tabular}

$$
\begin{aligned}
& S S_{\text {Factor }}=\frac{n \times r}{L} \sum_{k=1}^{L}\left(\overline{y_{k}}-\overline{\bar{y}}\right)^{2} \\
& S S_{\text {error }}=S S_{\text {total }}-\sum_{i=1}^{n} S S_{\text {Factor }_{i}}
\end{aligned}
$$

where $S S_{\text {Total }}$ is the sum of squares for all variances; $y_{i j}$, is the ranked grade of an image for the $i^{\text {th }}$ group in the $j^{\text {th }}$ trial of one specific phantom weight; $r$ is the total number of trial repeats in each group (in this study, $r=3 \times 3=9$, since the same gauge image was ranked for three rounds by three reviewers). Besides, $\overline{\bar{y}}$ is the average of all ranked values from the prompt phantom image (i.e., the theoretical value is 9.5). Parameter $S S_{\text {Factor }}$ is the sum of squares according to the specific factor, and $\overline{y_{k}}$ is the mean ranked value assigned to specific factor. Constants $L$ or $n$ is the numbers of levels assigned to the factor or to all groups. In this study, $n=18$ and $\mathrm{L}$ and $n=2$ or 3 . Finally, $S S_{\text {error }}$ is the sum of squared random errors.

Table 5 presents a significantly different distribution of $S S_{\text {Factor }}$ at various phantom weights: parameter $S S_{\text {error }}$ in the $70 \mathrm{~kg}$ phantom analysis reached $40 \%$, indicating an enormous discrepancy in viewers' ranking results, whereas its values in other weights were $7.8 \%$ for $50 \mathrm{~kg}$ and $20 \%$ for $90 \mathrm{~kg}$. This happens because the CTA scan images of 18 groups for the $70 \mathrm{~kg}$ phantom have a similar quality to be ranked (cf. Table 3); thus, the results for $70 \mathrm{~kg}$ are more controversial than the other two. It becomes difficult to improve the image quality and follow the quality assurance guideline since different radiologists have their interpretations of the imaging quality. However, CTA scan images are ranked easier at lower $(50 \mathrm{~kg})$ or larger $\left(90 \mathrm{~kg}\right.$ ) phantom weights to ensure a similar quality, and $S S_{\text {error }}$ drops significantly (cf. Table 5). Nevertheless, the dominant factors also fluctuate at different phantom weights. The only unchanged one is factor $\mathrm{F}$ (reconstruction filter). Factor $\mathrm{A}(\mathrm{kVp})$ is valid for 50 and $70 \mathrm{~kg}$; factor $\mathrm{B}(\mathrm{mAs})$ is valid for 70 and $90 \mathrm{~kg}$ and factor $\mathrm{C}$ (pitch) is valid only for $90 \mathrm{~kg}$. The routine preset of each factor has to be specifically considered in facing different phantom weights, and the cardiac CTA standard protocol also needs to be slightly revised according to various patients' weights.

\subsection{Sensitivity in the dynamic algorithm}

As the unique quality characteristic of Taguchi's dynamic algorithm, sensitivity can be customized and revised according to different perspectives. For most dynamic studies, high sensitivity is always 


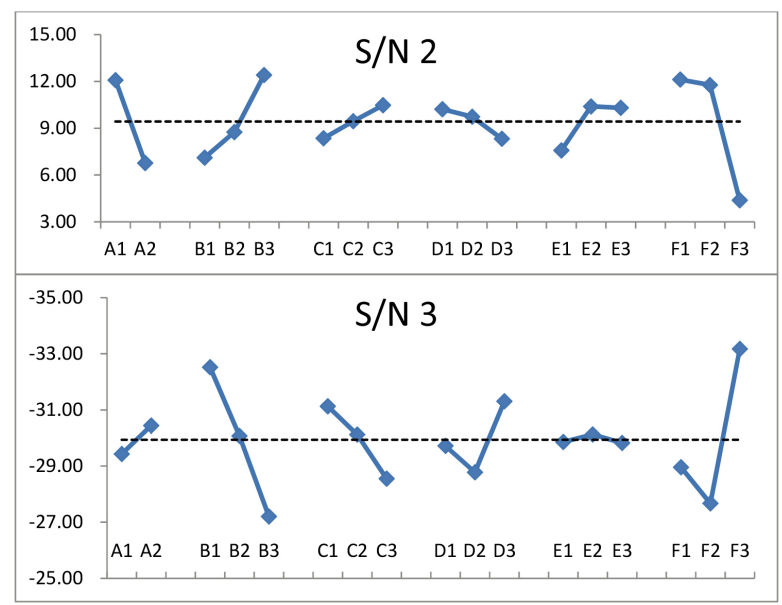

Fig. 6. The respective fish-bone plot of S/Ns constructed via Eqs (8) and (9).

preferable $[8,13,19]$. However, the sensitivity has a different interpretation in this study because the sensitivity implies the imaging quality versus various phantoms' weights. Thus, an insensible quality characteristic is preferable for radiologists in routine cardiac CTA scans. Accordingly, the parameter S/N $(\eta, \mathrm{dB})$ can be revised as follows:

$$
\begin{aligned}
& \mathrm{S} / \mathrm{N}_{2}(\mathrm{~dB})=-10 \log \left(\operatorname{stdev}^{2} / a v g^{2}\right), \\
& \mathrm{S} / \mathrm{N}_{3}(\mathrm{~dB})=-10 \log \left(s t d e v^{2} / \beta^{2}\right),
\end{aligned}
$$

where avg is the total average of all ranked scores from three viewers and three phantoms' weights (cf. Table 3, $3 \times 3 \times 3=27$ dataset), while $\beta$ has the same definition as in Eq. (1). The basic idea of Eq. (8) is to intensify the mathematical average without considering different phantom weights, i.e., to ignore the phantoms' weight change in the real CTA scan and average all terms under the same configuration. Meanwhile, Eq. (9) contains a conventional definition of the routine dynamic algorithm, which is inappropriate in our present scenario. Figure 6 reveals the respective fish-bone plot of S/Ns constructed via Eqs (8) and (9). As clearly illustrated, the variation trend of $\mathrm{S} / \mathrm{N}$ versus different factors may mislead the interpretation of dynamic algorithm and fail to achieve the required optimization.

\subsection{Dynamic algorithm superiority over the static one in Taguchi's suggestion}

In the typical static preset case, the original phantom configuration remains unchanged. In the dynamic one, the original phantom can be replaced by others of different sizes to match the real situation in routine work. The dynamic algorithm's unique sensitivity $(\beta)$ is a useful index to inspect the quality characteristic in robust designation, whereas the static one provides no such option. The sensitivity of the CTA image can also handle the real situation on a theoretically substantiated basis. In contrast, when the standard static algorithm is used, the ranked scores have to be summed and averaged altogether, although they were ranked under various phantom weights. Therefore, the $\mathrm{S} / \mathrm{N}_{2}$ value derived via Eq. (8) (cf. Fig. 6) may be the only option, so a possible misinterpretation of the $\mathrm{S} / \mathrm{N}_{2}$ fish-bone plot might yield an irrational solution, which further verification would be useless. For instance, the false optimal suggestion would be A1 (100 kVp), B3 (800 mAs), C3 (pitch $0.200 \mathrm{~mm}$ ), D1 (FOV $220 \mathrm{~mm}$ ), E2 (iDose 4), and F1 (filter XCC, whereas the exact optimal combination should be A1 (100 kVp), B1 (600 mAs), C3 (pitch $0.200 \mathrm{~mm})$, D3 (FOV $280 \mathrm{~mm}$ ), E3 (iDose 5), and F3 (filter XCA). Even the most dominant factor F (reconstruction filter) had an inappropriate setting (XCA instead of XCC). 


\section{Conclusions}

The MDD of cardiac CT angiography (CTA) was optimized using three (50, 70, and $90 \mathrm{~kg}$ ) PMMA phantoms and a V-shaped line gauge according to Taguchi's dynamic algorithm. As the unique feature of the dynamic algorithm, sensitivity was redefined to satisfy the preset scenario, i.e., an insensible quality characteristic was favored in facing various patients' weights for routine cardiac CTA diagnosis. Eighteen combinations of six factors were organized according to Taguchi's optimization analysis, whereas the dynamic algorithm was realized by changing the phantom sizes/weights to derive the sensitivity. A revised $\mathrm{S} / \mathrm{N}(\mathrm{dB})$ parameter was adopted to find the optimal factor combination of cardiac CTA to have the finest MDD of V-shaped line gauge. The results were A1 (100 kVp), B1 (600 mAs), C3 (pitch $0.200 \mathrm{~mm})$, D3 (FOV $280 \mathrm{~mm}$ ), E3 (iDose 5), and F3 (filter XCA). The derived MDD values were 2.15, 2.32, and $1.87 \mathrm{~mm}$ for 50, 70, and $90 \mathrm{~kg}$ PMMA phantoms. The static and dynamic optimization algorithms were compared, and the latter's superiority was substantiated.

\section{Acknowledgments}

The authors highly appreciate the financial support of this study by the Ministry of Science and Technology of the Republic of China (contract no. MOST 110-2221-E-166-001) and the Taichung Armed Forces General Hospital in Taiwan (contract no. TCAFGH-D-110034).

\section{Conflict of interest}

None to report.

\section{References}

[1] Pan LF, Erdene E, Pan LK. Optimization of the imaging quality of 64-slice CT acquisition protocol using Taguchi analysis: A phantom study, Bio-Medical Materials and Engineering, 2015; 26: s1651-1658.

[2] Kittipayak S, Pan LF, Chiang FT, et al. The optimization of the single photon emission computed tomography image quality via taguchi analysis: A feasibility study of a V-shaped phantom, J. Medical Imaging and Health Informatics, 2017; 7(1): 143-148.

[3] Pan LF, Wu KY, Chen KL, et al. Taguchi method-based optimization of the minimum detectable difference of a cardiac X-ray imaging system using a precise line pair gauge, JMMB, 2019; 19(7): 1940030.

[4] Peng BR, Kittipayak S, Pan LF, et al. Optimizing the minimum detectable difference of computed tomography scanned images via the Taguchi analysis: A feasibility study with an indigenous hepatic phantom and a line group gauge, $J M M B$, 2019; 19(8): 1940048.

[5] Lee TM, Lin CC, Peng BR, et al. Integration of Taguchi analysis with phantom and innovative gauges: Optimization of the CT scan protocol for peripheral arterial occlusive disease (PAOD) syndrome, JMMB, 2020; 20(9): 2040005.

[6] Yeh DM, Chang PJ, Pan LK. The optimum Ga-67-citrate gamma camera imaging quality factors as first calculated and shown by the Taguchi's analysis, Hell J Nucl Med, 2013; 16(1): 25-32.

[7] Huang CK, Wu J, Cheng KY, et al. Optimization of Imaging parameters for SPECT scans of [99mTc]TRODAT-1 using taguchi analysis, PLOS ONE, 2015; 10(3): e011381. doi: 10.1371/journal.pone.0113817.

[8] Chen CY, Liu KC, Chen HH, et al. Optimizing the TLD-100 readout system for various radiotherapy beam doses using the Taguchi methodology, Applied Radiation Isotopes, 2010; 68: 481-488.

[9] Yeh DM, Wang TH, Pan LK. Evaluating the quality characteristics of TLD-100T and TLD-100H exposed to diagnostic X-rays and 64 multislice CT using Taguchi's quality loss function, Radiation Measurement, 2015; 80: 17-22.

[10] Chiang FT, Chu KH, Chen CY, et al. Taguchi's analysis to optimize descending aortography for patent ductus arteriosus, with clinical verification, Hellenic Journal of Nuclear Medicine, 2016; 19(2): 118-123. 
[11] Chen CY, Pan LF, Chiang FT, et al. Optimizing quality of digital mammographic imaging using Taguchi analysis with an ACR accreditation phantom, Bioengineered, 2016; 7(4): 226-234.

[12] Pan LF, Chu KH, Sher HF, et al. Optimizing left anterior oblique (LAO) caudal imaging in coronary angiography using the Taguchi method: A phantom study with clinical verification, The International Journal of Cardiovascular Imaging, 2017; 33(9): 1287-1295.

[13] Pan LF, Le Y, Yen YC, et al. Optimizing the TLD-100H readout system under various radioactive I-131 doses via the revised Taguchi dynamic quality loss function, $J M M B, 2020 ; 20(10): 2040024$.

[14] Schoepf UJ. CT of the Heart, $2^{\text {nd }}$ ed. Humana Press. USA, ISBN: 978-1-60327-236-0.

[15] Roy RK. A primer on the Taguchi method, $2^{\text {nd }}$ ed. Society of Manufacturing Engineering, USA ISBN 13:978-0-87263864-8.

[16] White DR, Buckland-Wright JC, Griffith RV, et al. Phantoms and computational models in therapy, diagnosis and protection, ICRU Report 48, 1992; 25(1). doi: 10.1093/jicru/os25.1.Report48.

[17] Kenny DA, Mannetti L, Pierro A, et al. The statistical analysis of data from small groups, J Personal Soc Psychol, 2002; 83(1): 126-137.

[18] Chang CF, Chen CF, Wang TH, et al. Semi-quantification of the minimum detectable difference of gamma camera SPET for four radionuclides via an innovative PMMA phantom with a V-shaped slit: Interpretation of a feasibility study, HJNM, 2019; 22(1): 7-14.

[19] Kuo HN, Yi JE, Chen CH, et al. Taguchi dynamic analysis application to computer tomography number-mass density linear dependence optimization, Computer Assisted Surgery, 2017; 22(s1): 45-53. 